\title{
Effects of dietary Hermetia illucens meal inclusion on cecal microbiota and small intestinal mucin dynamics and infiltration with immune cells of weaned piglets
}

Ilaria Biasato ${ }^{1}$, Ilario Ferrocino ${ }^{1}$, Elena Colombino ${ }^{2}$, Francesco Gai ${ }^{3}$, Achille Schiavone ${ }^{2,3^{*}}$ (D, Luca Cocolin ${ }^{1}$, Valeria Vincenti ${ }^{2}$, Maria Teresa Capucchio ${ }^{2,3}$ and Laura Gasco ${ }^{1}$

\begin{abstract}
Background: The constant interaction between diet and intestinal barrier has a crucial role in determining gut health in pigs. Hermetia illucens $(\mathrm{HI})$ meal (that represents a promising, alternative feed ingredient for production animals) has recently been demonstrated to influence colonic microbiota, bacterial metabolite profile and mucosal immune status of pigs, but no data about modulation of gut mucin dynamics are currently available. The present study evaluated the effects of dietary $\mathrm{HI}$ meal inclusion on the small intestinal mucin composition of piglets, as well as providing insights into the cecal microbiota and the mucosal infiltration with immune cells.

Results: A total of 48 weaned piglets were randomly allotted to 3 dietary treatments (control diet [C] and 5\% or $10 \% \mathrm{HI}$ meal inclusion [HI5 and HI10], with 4 replicate boxes/treatment and 4 animals/box) and slaughtered after 61 days of trial (3 animals/box, 12 piglets/diet). The cecal microbiota assessment by $16 \mathrm{~S}$ rRNA amplicon based sequencing showed higher beta diversity in the piglets fed the HI-based diets than the $C(P<0.001)$. Furthermore, the HI-fed animals showed increased abundance of Blautia, Chlamydia, Coprococcus, Eubacterium, Prevotella, Roseburia, unclassified members of Ruminococcaceae, Ruminococcus and Staphylococcus when compared to the C group (FDR $<0.05)$. The gut of the piglets fed the HI-based diets showed greater neutral mucin percentage than the $C(P<0.05)$, with the intestinal neutral mucins of the HI-fed animals being also higher than the sialomucins and the sulfomucins found in the gut of the $C$ group $(P<0.05)$. Furthermore, the piglets fed the HI-based diets displayed lower histological scores in the jejunum than the other gut segments (ileum [HI5] or ileum and duodenum [HI10], $P<0.05)$.

Conclusions: Dietary HI meal utilization positively influenced the cecal microbiota and the small intestinal mucin dynamics of the piglets in terms of selection of potentially beneficial bacteria and preservation of mature mucin secretory architecture, without determining the development of gut inflammation. These findings further confirm the suitability of including insect meal in swine diets.
\end{abstract}

Keywords: $16 \mathrm{~S}$ rRNA, Gut health, Hermetia illucens L., Histology, Insect meal, Microbiota, Mucin, Pig

\footnotetext{
* Correspondence: achille.schiavone@unito.it

2Department of Veterinary Sciences, University of Turin, Largo Paolo Braccini

2, 10095 Grugliasco, TO, Italy

${ }^{3}$ Institute of Science of Food Production, National Research Council, Largo

Paolo Braccini 2, 10095 Grugliasco, TO, Italy

Full list of author information is available at the end of the article
}

C The Author(s). 2020 Open Access This article is licensed under a Creative Commons Attribution 4.0 International License, which permits use, sharing, adaptation, distribution and reproduction in any medium or format, as long as you give appropriate credit to the original author(s) and the source, provide a link to the Creative Commons licence, and indicate if changes were made. The images or other third party material in this article are included in the article's Creative Commons licence, unless indicated otherwise in a credit line to the material. If material is not included in the article's Creative Commons licence and your intended use is not permitted by statutory regulation or exceeds the permitted use, you will need to obtain permission directly from the copyright holder. To view a copy of this licence, visit http://creativecommons.org/licenses/by/4.0/. The Creative Commons Public Domain Dedication waiver (http://creativecommons.org/publicdomain/zero/1.0/) applies to the data made available in this article, unless otherwise stated in a credit line to the data. 


\section{Introduction}

In the swine industry, which represents one of the major meat source for humans [1], the feed efficiency is a particularly critical aspect, since feed accounts for the majority of the total production costs [2]. Apart from animal genetics, disease, and production management, diet is considered one of the main factors influencing the feed efficiency in pigs [3]. The crucial role of the diet is related to its constant interaction with the gut barrier, which is constituted by microbiota and their products, mucus layers, host-derived antimicrobial compounds, epithelium, and underlying immune tissue [4]. In particular, researchers have focused their attention on the intestinal microbiota and mucin composition, as they can be widely affected by dietary modifications $[5,6]$. The gut microbiota has a key impact on host metabolism, immune functions and physiology, thus exerting a significant influence on gut and systemic health, as well as nutrient processing and energy harvesting [7]. The intestinal commensal microbes also depend on diet and mucus for nutrient and energy source and binding sites, respectively [8]. Furthermore, the gut microbiota and microbial products are capable of modulating the mucin synthesis and secretion, both through the direct activation of several signalling cascades and the indirect generation of bioactive factors by the gut mucosa [6]. Mucins are multifunctional glycoproteins that compose the gut mucus layer and are mainly involved in the intestinal protection and nutrient digestion and absorption [9]. Therefore, investigating both the gut microbiota and the mucins seems to be fundamental in finding effective strategies for the improvement of pig intestinal health and feed efficiency, especially when a novel feed ingredient is tested. Another important aspect to consider is that piglets, especially in the postweaning period, are under great environmental pressure, thus causing a decline in their immune function and, in turn, development of gut inflammation. As a consequence, histological analysis of the gut may also provide useful information about the health status of the intestinal barrier [10].

Within the animal production scenario, the use of insects as alternative feed ingredients has rapidly become a consolidated reality, not only due to their remarkable nutritive properties and advantageous rearing characteristics [11], but also to their potential ability to modulate the intestinal microbiota with positive effects on animal health [12]. Among the insect species investigated for animal feeding purposes, Hermetia illucens (HI) has recently gained the greatest attention in pig farming [13-15]. In particular, HI prepupa and larva meals proved to be highly digestible and safe for weaned piglets, with no negative influence being observed on animal health and performance and gut mucosal morphology [16, 17]. These studies establish the bases of the authorization to feed the insect proteins to pigs, which is currently prohibited by the Regulation No 999/2001 [18]. Furthermore, significant in vitro gut antimicrobial effects against $D$-streptococci (opportunistic pathogens) have been ascribed to HI prepupa fat utilization, with the authors attributing these positive effects to its high content of lauric acid [16]. Yu et al. [19] recently reported that dietary HI larva meal inclusion may enhance the colonic mucosal immune homeostasis of finishing pigs via positively altering the bacterial composition and their metabolites, thus confirming the antimicrobial properties of HI previously highlighted. However, if the study of intestinal microbiota in insect-fed pigs has made significant progresses, data about gut mucin composition modulation by insect meal utilization are still lacking.

Based on the above reported background, the present study aims to evaluate the effects of dietary HI meal inclusion on gut microbiota, mucin composition and infiltration with immune cells of weaned piglets.

\section{Materials and methods}

\section{Piglets and experimental design}

The experimental design of the present study is reported by Biasato et al. [17]. In order to give a brief summary, 48 weaned piglets ( $20 \pm 1$ days of age, initial body weight: $6.1 \pm 0.16 \mathrm{~kg}$ ) were randomly distributed to four isoenergetic and isonitrogenous dietary treatments. Each diet was offered to 4 replicate pens (boxes) of 4 piglets each. Corn meal-, barley meal-, and soybean meal-based diet was used as the control diet (C), while the two experimental dietary treatments (indicated as $\mathrm{HI} 5$ and HI10) were obtained by including $5 \%$ and $10 \%$ partially defatted HI larva meal (Hermetia Baruth GmbHo. KG, Baruth / Mark, Germany), respectively, as partial replacements of the soybean meal. The chemical composition of the $\mathrm{HI}$ larva meal was as follows: $947.4 \mathrm{~g} / \mathrm{kg}$ dry matter, 559 $\mathrm{g} / \mathrm{kg}$ crude protein, and $85 \mathrm{~g} / \mathrm{kg}$ ether extract, as fed. Details of the diets are shown in Table S1. The growth performance of the piglets were also evaluated throughout the experimental trial, as reported in details by Biasato et al. [17]. Briefly, no overall significant differences were observed for growth performance, except for the average daily feed intake of the second feeding phase showing a linear response to increasing HI larva meal levels. The experimental period lasted 61 days.

\section{Intestinal sampling and processing}

A total of twelve piglets per treatment (three animals per box) were randomly selected and slaughtered in a commercial abattoir at the end of the experimental trial. 
The animals were stunned by electrocution and exsanguinated. Cecal content was collected into sterile plastic tubes that were promptly refrigerated (for a maximum of $2 \mathrm{~h}$ ) and frozen at $-80^{\circ} \mathrm{C}$ until DNA extraction. Intestinal segment samples (approximately $5 \mathrm{~cm}$ in length) of duodenum, jejunum and ileum were excised and flushed with $0.9 \%$ saline to remove all the content. The collected segments of intestine were the tract after the pylorus (duodenum), the mid jejunum (jejunum) and the tract before the ileocecal junction (ileum). Gut segments were fixed in $10 \%$ buffered formalin solution for histological examination and histochemical staining. Tissues were routinely embedded in paraffin wax blocks, sectioned at $5 \mu \mathrm{m}$ thickness and mounted on glass slides.

\section{DNA extraction and sequencing}

The nucleic acid was extracted by pooling the cecal content from three slaughtered piglets per box (four pools per feeding group). The total genomic DNA (gDNA) was extracted from the samples using the RNeasy Power Microbiome KIT (Qiagen, Milan, Italy) following the manufacturer's instructions. One microliter of RNase (Illumina Inc., San Diego, CA) was added to digest RNA in the DNA samples with an incubation of $1 \mathrm{~h}$ at $37^{\circ} \mathrm{C}$. The DNA was quantified using the NanoDrop and standardized at $5 \mathrm{ng} / \mu \mathrm{L}$. The gDNA was used to assess the microbiota by the amplification of the V3-V4 region of the 16S rRNA gene [20]. The PCR products were to the illumina metagenomic pipeline. Sequencing was performed with a MiSeq Illumina instrument (Illumina) with V3 chemistry and generated $250 \mathrm{bp}$ paired-end reads, following the manufacturer's instructions.

\section{Histochemical staining}

The paraffin-embedded intestinal sections of the piglets were also submitted to a triple staining that demonstrated the different mucin subtypes, according to Rieger et al. [21]. Firstly, sections were stained with the periodic acid-Schiff, which identified the neutral mucins in magenta. The second staining step was the Alcian blue $\mathrm{pH} 2.5$, which stained the sialomucins in turquoise. Finally, sections were stained with the high iron diamine, which identified the sulfomucins in brownish-purple to black [21].

\section{Mucin staining quantification}

One slide per histochemical staining for each intestinal segment was examined by means of light microscopy. Five randomly selected high power fields per each slide were captured with a Nikon DS-Fi1 digital camera coupled to a Zeiss Axiophot microscope using a 20× objective lens and NIS-Elements $\mathrm{F}$ software was used for image capturing. Mucin staining quantification was then performed by Image ${ }^{\bullet}$-Pro Plus software. The presence of mucins was estimated as the percentage of the gut mucosal area (covering both the crypts and the villi) that was positive for the histochemical staining, as previously described [22]. In particular, mucins were automatically identified by means of pixel classification [21].

\section{Histological examination}

The paraffin-embedded intestinal sections of the piglets were submitted to the Haematoxylin \& Eosin (HE) staining to evaluate the gut infiltration with immune cells, as reported in details by Biasato et al. [17]. One slide per HE section was examined by means of light microscopy. For each gut segment, the mucosa and the submucosa were separately assessed for the immune cell infiltrates (mucosa and submucosa) and the gut-associated lymphoid tissue (GALT) activation (submucosa) using a semiquantitative scoring system from 0 (absence of alterations) to 3 (severe alterations). The total score of each gut segment was then obtained by adding the mucosa and the submucosa scores.

\section{Bioinformatics and statistical analysis}

Paired-end reads were first assembled with FLASH [23] and quality filtered (at Phred < Q20) using QIIME 1.9.0 software [24], and the recently described pipeline was adopted [25]. Briefly, Operational Taxonomic Units (OTUs) were picked at $97 \%$ of similarity and centroids sequences were used to assign taxonomy using the Greengenes 16S rRNA gene database (version 2013). Alpha diversity indices were calculated using the diversity function of the vegan package [26] and analyzed using the pairwise Wilcoxon rank sum test to assess the differences between the dietary treatments. A filtered OTU table was generated at $0.1 \%$ abundance in at least 2 samples through QIIME. The table was then used to build the Principal component analysis (PCA). OTU table displayed the highest taxonomy resolution. Weighted UniFrac distance matrices and OTU table were used to perform Adonis and ANOSIM statistical tests in $\mathrm{R}$ environment. Pairwise Kruskal-Wallis test was used to find significant differences in microbial taxa abundance among the dietary treatments. $P$ values were adjusted for multiple testing and a false discovery rate $($ FDR $)<0.05$ considered as significant.

The statistical analysis of the histochemical and the histological data was performed using IBM SPSS Statistics V20.0.0 software. In relation to the histochemical data, a generalized linear model (GLM) was fitted to allow the mean gut mucin staining percentages to depend on linear predictors such as diet, mucin type, intestinal segment and their corresponding interactions through a gamma probability distribution with a nonlinear link function (log). The piglet and the pen within treatment effect were also included in the GLM as the 
repeated factors. Differently, the histological data were tested by fitting a GLM that allowed the total gut scores to depend on linear predictors such as diet, intestinal segment and their interaction through a negative binomial response probability distribution with a nonlinear link function $(\log )$. The piglet and the pen within treatment effect were also herein included in the GLM as the repeated factors. A hybrid method for parameter estimation was used for both the GLMs and a type III analysis with Wald chi-square test was applied to assess the model effects. All the obtained results were expressed as least squares means and SEM and the interactions between the factor levels were evaluated by pairwise comparisons. $P$ values $<0.05$ were considered statistically significant.

\section{Results}

\section{Cecal microbiota characterization}

A total of 916,380 raw reads $(2 \times 250 \mathrm{bp})$ were obtained after sequencing. After joint and quality filtering, a total of 858,032 reads passed the filters applied through QIIME, with an average value of 71,502 reads/sample and a median sequence length of $465 \mathrm{bp}$. The rarefaction analysis and the Good's coverage revealed a satisfactory coverage for all the samples (average Good's coverage of 98\%, Table S2). Dietary HI larva meal inclusion did not affect the alpha diversity indices (PD Whole Tree, Chao1, observed species richness and Shannon, Table S2, $P>0.05$ ), whereas ADONIS and Anosim statistical tests based on Weighted UniFrac distance matrix showed significant differences among the dietary treatments $(P<0.001)$. In particular, the PCA showed a clear separation between the $\mathrm{HI}$ samples and those from the C-fed piglets (Fig. 1).

With regards to the most abundant OTUs, both the $\mathrm{C}$ - and the HI-fed groups showed Firmicutes, Proteobacteria and Bacteroidetes as predominant phyla in their cecal microbiota (Fig. 2, Table S3), as well as Actinobacillus, unclassified members (U. m.) of Clostridiaceae, U. m. of Enterobacteriaceae, Lactobacillus and Streptococcus as predominant genera (Fig. 2, Table S3).

Comparing the relative abundance of the main OTUs across the samples, the piglets fed the HI-based diets showed increased abundance of Blautia, Chlamydia, Coprococcus, Eubacterium, Prevotella, Roseburia, U. m. of Ruminococcaceae, Ruminococcus and Staphylococcus when compared to the $\mathrm{C}$ group (Fig. 3, FDR $<0.05$ ).

\section{Intestinal mucin composition}

The mucin staining percentages in the gut of the piglets significantly depended on the mucin type $(P<0.001)$, the gut segment $(P<0.001)$, and interaction between the diet and the mucin type $(P<0.05)$. On the contrary, there was no significant influence of dietary $\mathrm{HI}$ meal inclusion $(\mathrm{C}=7.64 \pm 0.36$; $\mathrm{HI} 5=8.66 \pm 0.58 ; \mathrm{HI} 10=8.17 \pm 0.30)$ on the histochemical findings $(P>0.05)$. No significant interactions between the diet and the gut segment, the mucin type and the gut segment, and the diet, the gut segment and the mucin type $(P<0.05)$ were also identified (Table 1). In particular, the intestine showed higher

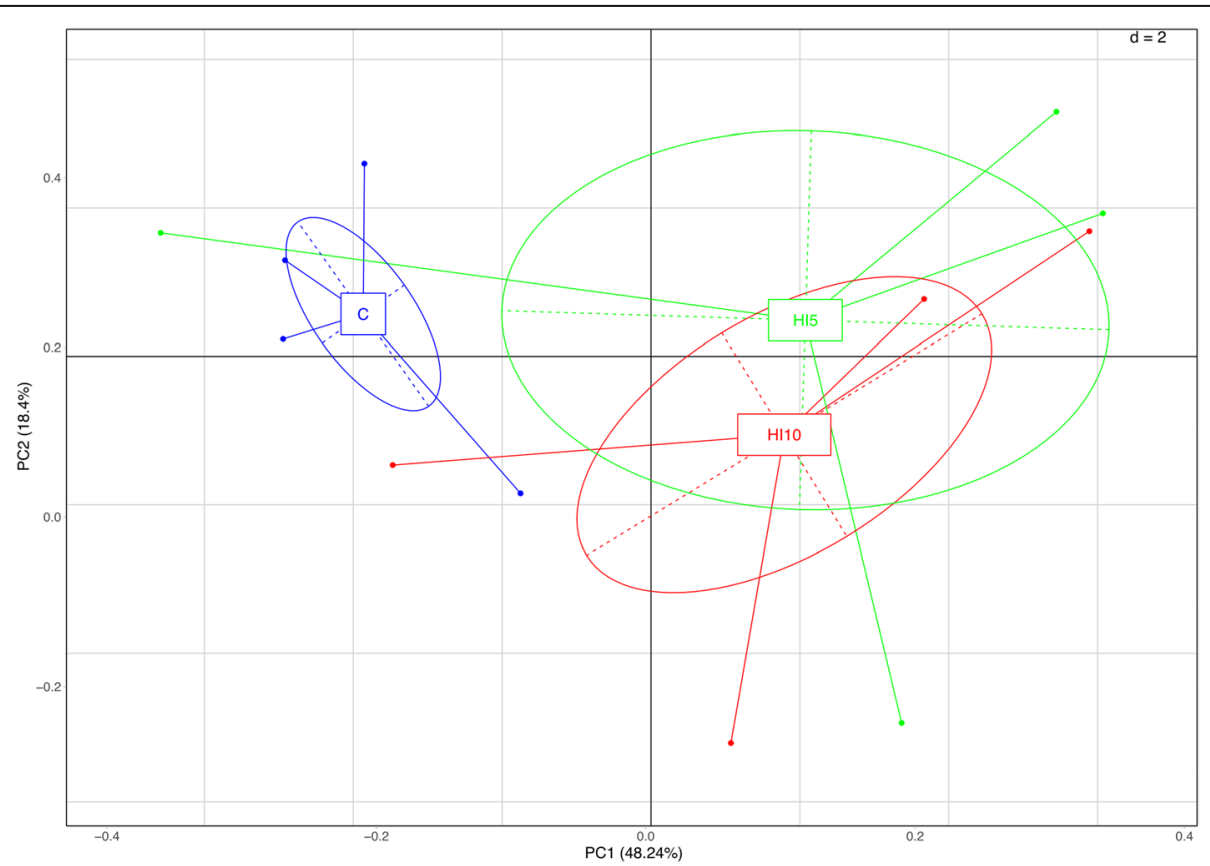

Fig. 1 Bacterial community composition (weighted UniFrac beta diversity, PCA plots) in cecal samples of piglets fed control (C) and 5\% (HI5) and 10\% (H110) inclusion levels of Hermetia illucens meal diets 

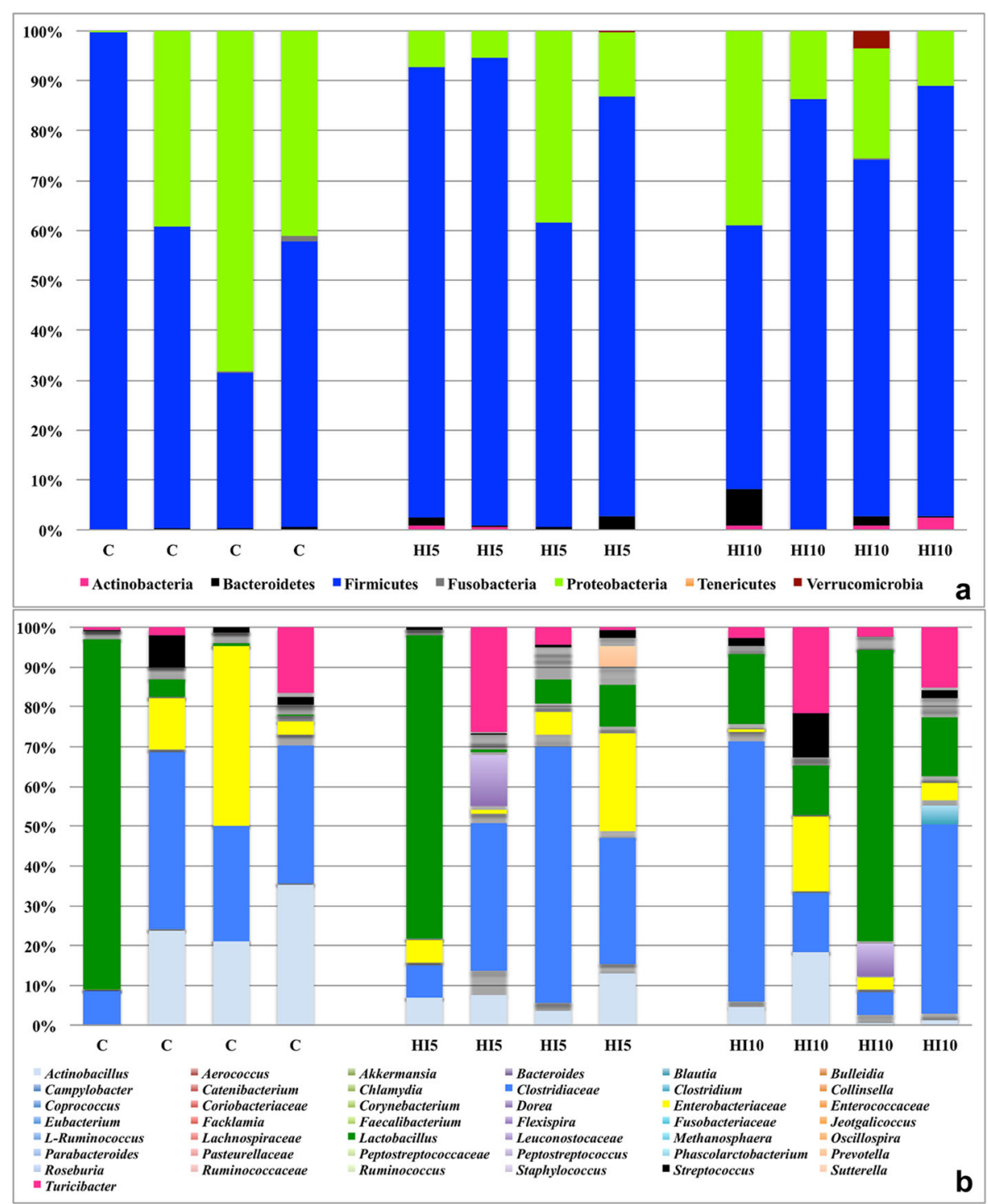

Fig. 2 Relative abundance of the main bacterial phyla (a) and genera (b) in cecal samples of piglets fed control (C) and 5\% (HI5) and 10\% (HI10) inclusion levels of Hermetia illucens meal diets. Graph bar indicate the 4 replicate boxes per each dietary treatment

neutral mucin staining percentage when compared to the other mucin subtypes $(P<0.001)$, with the sialomucins being also greater than the sulfomucins $(P<0.001$, Fig. 4). Furthermore, higher mucin staining percentage was identified in the duodenum and the ileum in comparison with the jejunum $(P<0.001$, Figs. 4 and 5$)$. The gut of the HI-fed piglets also showed greater neutral mucin staining percentage than the $C$ group $(P<0.05)$, with the intestinal neutral mucins of the $\mathrm{HI}$ animals being also higher than the sialomucins and the sulfomucins found in the gut of the $C$ group $(P<0.05$, Fig. 6).

\section{Intestinal infiltration with immune cells}

The histological scores in the gut of the piglets significantly depended on the gut segment $(P<0.001)$, while no significant influence of dietary HI meal inclusion $(\mathrm{C}=2.66 \pm 0.38 ; \quad \mathrm{HI} 5=2.71 \pm 0.32 ; \quad \mathrm{HI} 10=3.16 \pm 0.52)$ was observed $(P>0.05)$. The histological scores were also not significantly affected by the interaction between the diet and the gut segment $(P>0.05$, Table 1$)$. In particular, the ileum showed higher infiltration with immune cells when compared to the other gut segments $(P<0.001$, Fig. 7$)$.

\section{Discussion}

Cecal microbiota characterization

Firmicutes, Proteobacteria and Bacteroidetes represented the dominant bacterial phyla in both the C- and HI-fed piglets of the present study. These findings overall agree with the previous researches that identified Firmicutes 


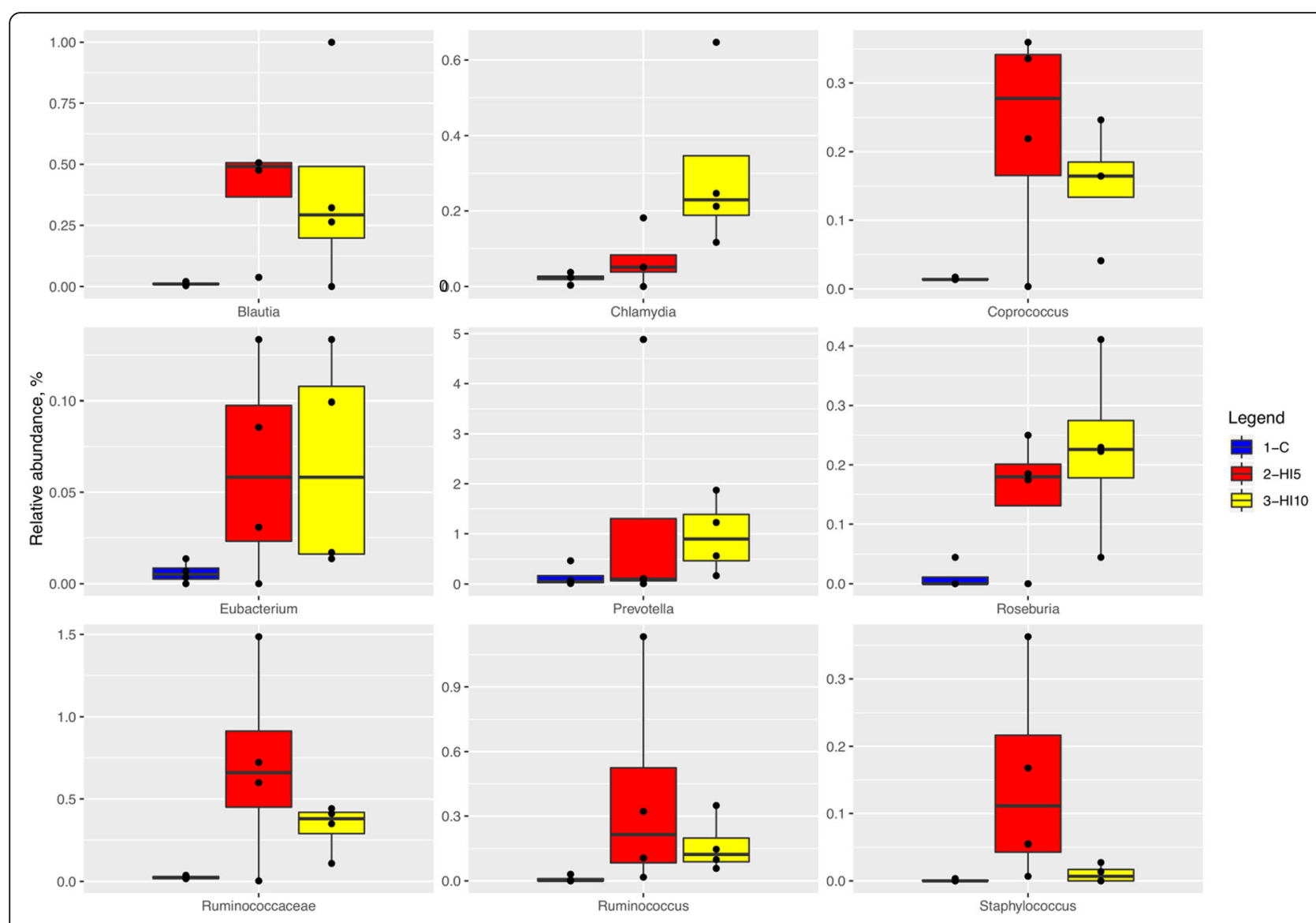

Fig. 3 Relative abundance at phylum level of differentially abundant OTUs in cecal samples piglets fed control (C) and 5\% (HI5) and 10\% (HI10) inclusion levels of Hermetia illucens meal diets. Pairwise Kruskal-Wallis test, FDR $<0.05$

Table 1 Effects of the different linear predictors on the histochemical findings and the histological scores in the gut of the piglets

\begin{tabular}{llll}
\hline Histochemical findings $^{\text {a }}$ & d.f. $^{\text {d }}$ & Chi-square & $P^{f}$ \\
\hline Diet $^{\mathrm{a}}$ & 2 & 2.614 & 0.271 \\
Mucin type $^{\mathrm{b}}$ & 2 & 53.724 & $<0.001$ \\
Gut segment $^{\mathrm{c}}$ & 2 & 34.164 & $<0.001$ \\
Diet $\times$ Mucin type & 4 & 12.216 & 0.016 \\
Diet $\times$ Gut segment & 4 & 1.017 & 0.907 \\
Mucin type $\times$ Gut segment & 4 & 7.878 & 0.096 \\
Diet $\times$ Mucin type $\times$ Gut segment & 8 & 11.507 & 0.175 \\
Histological scores & & & \\
$\quad$ Diet & 2 & 0.739 & 0.691 \\
$\quad$ Gut segment & 2 & 32.113 & $<0.001$ \\
$\quad$ Diet $\times$ Gut segment & 4 & 1.658 & 0.798 \\
\hline
\end{tabular}

${ }^{\mathrm{a}}$ Three dietary treatments: $\mathrm{C}=$ control; $\mathrm{HI} 5=5 \%$ inclusion level of Hermetia illucens; $\mathrm{HI} 10=10 \%$ inclusion level of Hermetia illucens

${ }^{\text {b}}$ Three types: neutral, acidic sialylated and acidic sulfated mucins

'Three gut segments: duodenum, jejunum and ileum

${ }^{\mathrm{d}}$ Degrees of freedom

fStatistical significance: $P<0.05$
[5, 27-29] as main bacterial phylum in the pig cecum, followed by Proteobacteria [27, 29] and Bacteroidetes [29]. In relation to the genera composition, Actinobacillus, Lactobacillus and Streptococcus, as well as members of Clostridiaceae and Enterobacteriaceae families, mainly colonized the cecal microbiota of the piglets fed either the $\mathrm{C}$ or the HI-based diets in the current research. These findings are also in agreement with the previous studies, which observed Lactobacillus [3, 5, 19, 27, 29], Streptococcus [5, 19, 27, 29] and Actinobacillus [29] as main bacterial genera in the cecal microbiota of pigs.

The microbial composition of the cecal digesta obtained from the piglets of the current research was significantly affected by dietary HI larva meal inclusion, as demonstrated by the increased beta diversity observed in the HI groups. This is in agreement with Yu et al. [19], who identified significant dissimilarities between the colonic microbiota of C- and HI-fed finishing pigs. These findings also confirm in swine species what previously reported in poultry, where insect meal utilization proved to be capable of creating a more diverse (and, in turn, stable) intestinal microbiota [25, 30, 31]. 


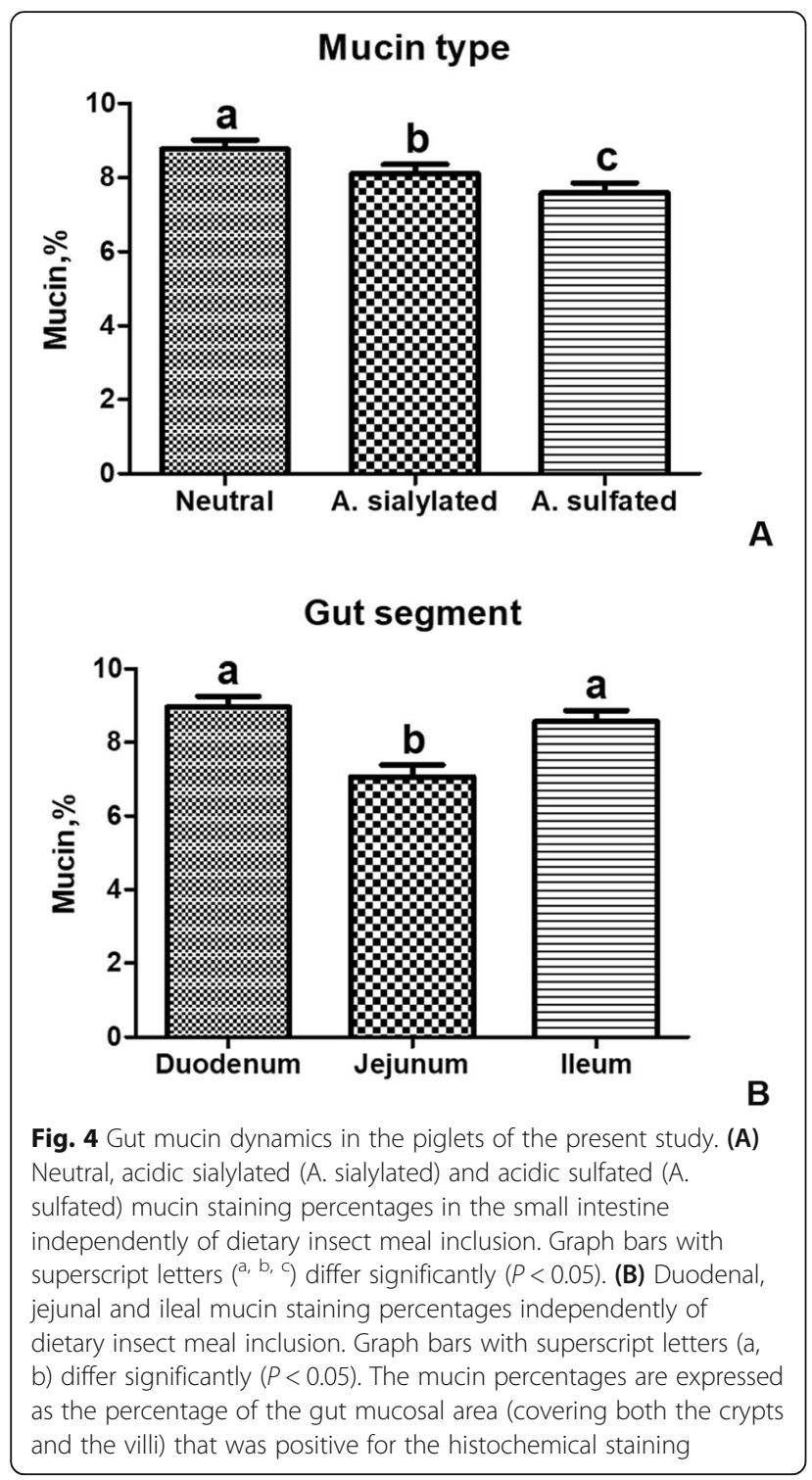

A specific signature at genus level was also observed in the cecal microbiota of the HI-fed piglets of the present study, where Blautia, Chlamydia, Coprococcus, Eubacterium, Prevotella, Roseburia, Ruminococcaceae, Ruminococcus and Staphylococcus were markedly predominant. Blautia [32], Coprococcus [33], Eubacterium [34], Prevotella [35-37], Roseburia [38], members of Ruminococcaceae [39, 40] and Ruminococcus [41, 42] are all taxon involved in polysaccharide degradation and fermentation, that boosted the production of short chain fatty acids (SCFAs) (mainly butyrate). The SCFAs have significant health benefits for the gut barrier [43], with butyrate being particularly essential for maintaining the intestinal metabolism [44], promoting the epithelial energy metabolism and stimulating the immune development [45]. Prevotella has also been reported to be involved in amino acid metabolism of host and positively influence the porcine intramuscular fat, which is considered an indicator for meat quality of pigs [37]. Furthermore, Ruminococcaceae family - that generally represents a core taxon with a relative abundance of $5 \%$ to $10 \%$ - is capable of improving the feed efficiency in pigs [3, 33]. Increase in SCFAs-producing bacteria, as well as SCFAs, has already been reported in both laying hens [30] and pigs [19] fed HI larva meal-based diets. These changes were attributed to the chitin content of the insect meal, which may serve as substrate for the gut microbiota, thus affecting either their composition or their microbial fermentation metabolites $[19,30]$. Despite no SCFAs detection having been performed in the present study, the analogous identification of SCFAsproducing bacteria allows to hypothesize a similar way of action of HI larva meal in the piglets' gut. Therefore, the increase in the above-mentioned bacterial taxa by $\mathrm{HI}$ larva meal utilization may have helped the piglets to maintain a healthy gut and show, consequently, similar growth performance to the $\mathrm{C}$ animals. Differently, in relation to the other increased OTUs observed in the HI-fed animals, Chlamydia and Staphylococcus genera comprises pathogenic bacteria, thus representing a potential negative finding. However, it is important to underline that the piglets fed the HI-based diets remained clinically healthy throughout the experimental trial and showed no significant alterations at the histological examination [17]. Since the growth performance were also overall unaffected by insect meal utilization, the positive increase in the SCFAs-producing bacteria could have mitigate this negative microbiota modulation.

\section{Intestinal mucin composition}

Independently of dietary HI meal inclusion, the small intestine of the piglets of the present study showed higher neutral mucin staining percentage than the other subtypes. The physiologic relevance of the distinct mucin subtypes has not been well understood yet, with data in pigs being also particularly scarce and rather conflicting [46]. Increase in neutral mucins during post-weaning has been suggested to be related to the physiological variation in villi and crypt depth, thus, in turn, affecting the goblet cell differentiation and normal maturation [47]. Indeed, mucins in the neonatal piglets are highly acidic $[48,49]$. Therefore, a predominance of neutral mucins is considered indicative of an increased intestinal maturity to facilitate the breakdown of complex carbohydrates [50]. As a confirmation of this aspect, Rieger et al. [21] recently observed higher staining percentage of neutral mucins (40\%) than sulfomucins $(8 \%)$ and sialomucins (2\%) in weaned piglets from different feeding trials.

Independently of insect meal utilization, the small intestine of the piglets of the current research displayed greater mucin staining percentage in the duodenum and 


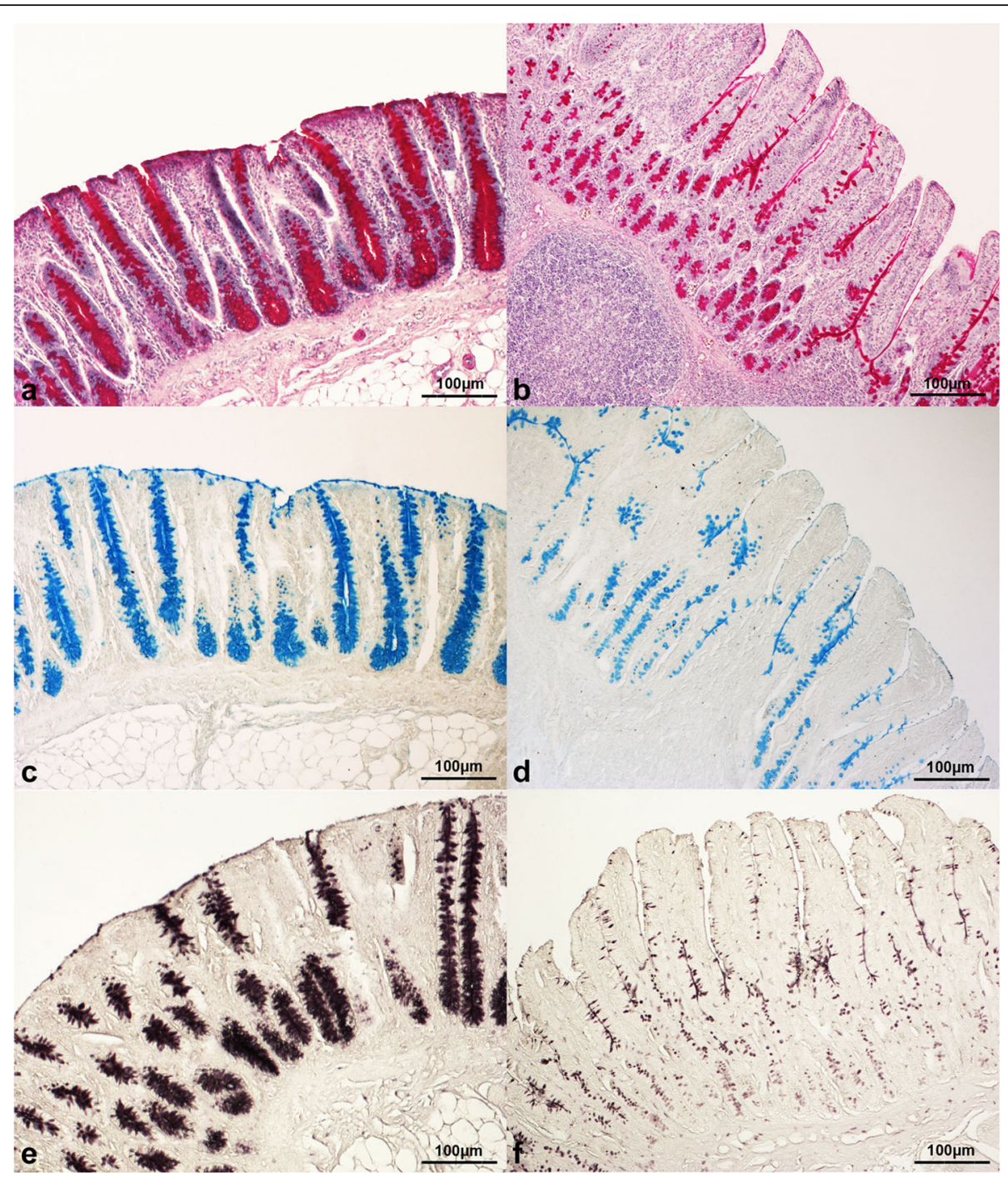

Fig. 5 Histological pictures of ileal (a, $\mathbf{c}, \mathbf{e})$ and jejunal (b, d, f) samples stained with (a, b) periodic-acid Schiff (20x magnification), (c, d) Alcian Blue $\mathrm{pH} 2.5$ (20x magnification) and (e, $\mathbf{f})$ high iron diamine (20x magnification) from the piglets of the present study. Ileal samples show higher mucin staining intensity than the jejunal ones

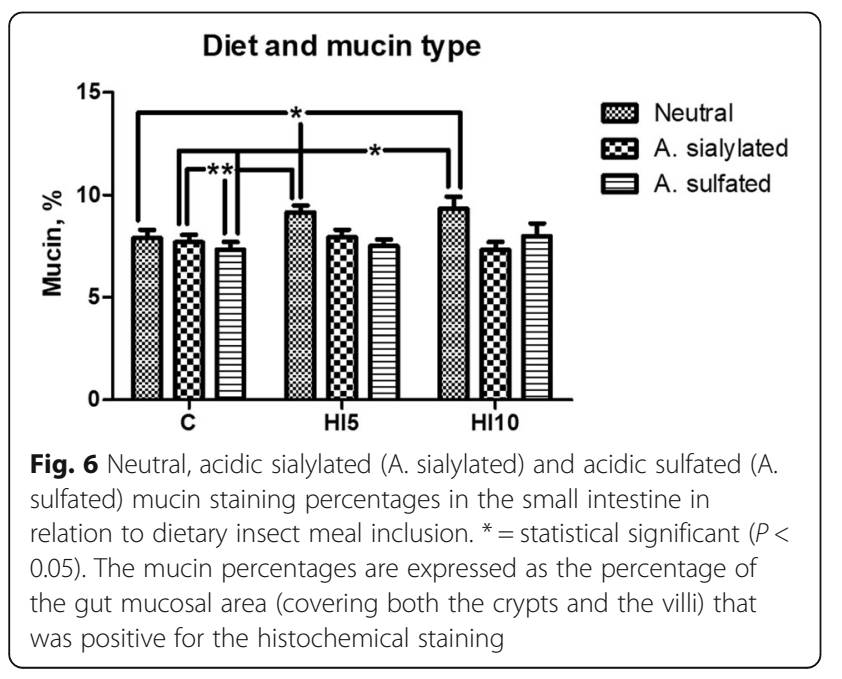

the ileum when compared to the jejunum. The mucin dynamics in the distinct segments of pig intestine have not been elucidated yet, since very limited studies have focused their attention on multiple intestinal tracts [21, 51]. However, each gut segment has its own specific characteristics that could explain the different histochemical findings. Indeed, the secretion of mucins in the duodenum has been related to the need of neutralizing of the acidic $\mathrm{pH}$ of the entering gastric juices [52]. Furthermore, since several pig pathogens (i.e., Salmonella Typhimurium and Lawsonia intracellularis) mainly colonize the ileal mucosa, the mucin production may be particularly useful as protective strategy. Therefore, the predominant staining of mucins observed in the duodenum and the ileum may be attributed to their different anatomy and physiology. 


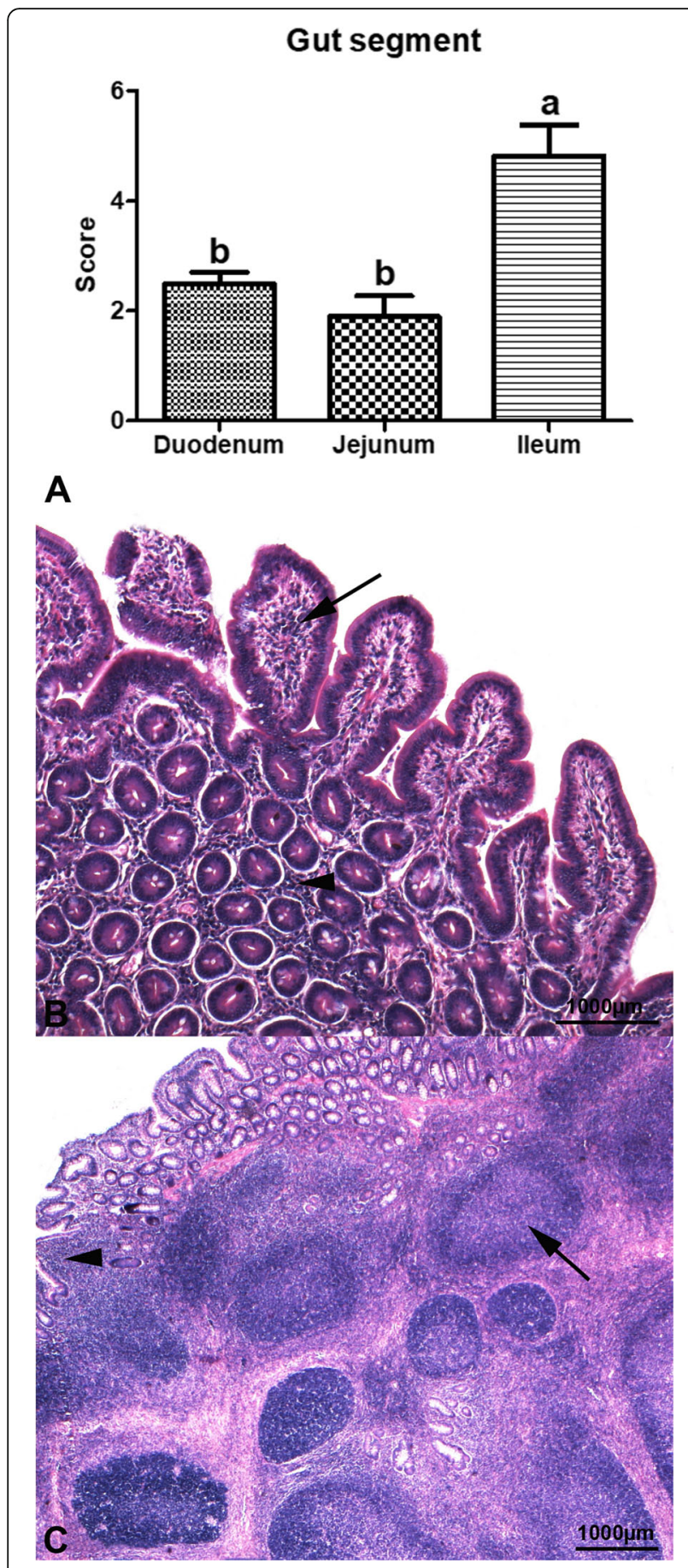

Fig. 7 Gut infiltration with immune cells in the piglets of the present study. (A) Histological scores in the small intestine independently of dietary insect meal inclusion. Graph bars with superscript letters $(\mathrm{a}, \mathrm{b})$ differ significantly $(P<0.05)$. (B) HI5 group. Mild, multifocal mucosal (arrow) and submucosal (arrowhead) lymphoplasmacytic infiltration is observed in the jejunum. Haematoxylin \& Eosin stain, $5 x$

magnification). (C) C group. The ileum shows severe, focal mucosal and submucosal lymphoplasmacytic infiltration (arrowhead), as well as severe, multifocal Gut-Associated Lymphoid Tissue (GALT) activation (arrow). Haematoxylin \& Eosin stain, $2.5 \times$ magnification
Interestingly, the piglets fed HI-based diets of the present study showed higher neutral mucin staining percentage in comparison with all the mucin subtypes of the $\mathrm{C}$ group. This could have positive implications, since the production of neutral mucins has been suggested to serve as a protective mechanism against invasion by pathogenic bacteria [53, 54]. Furthermore, as the identification of the neutral mucins is predominant in the mature gut, insect meal utilization may contribute to the preservation of a well-developed mucin secretory architecture. However, it is important to consider that both the gut sampling and the fixation methods may have caused the loss of most of the non-tissue mucins, thus representing a limitation of quantifying the gut mucins by histochemical analysis.

\section{Intestinal infiltration with immune cells}

The present study provides a useful, easy-to-use, histological semiquantitative scoring system to give reliable information about the gut infiltration with immune cells in piglets. Biasato et al. [17] previously observed gut mucosal/submucosal lymphoplasmacytic or eosinophilic infiltrates - with or without GALT activation - in both the $\mathrm{C}$ - and the HI-fed piglets of the present study, attributing these alterations to the feeding practices and reporting no significant effect of insect meal utilization on the mean intestinal histological scores. The novel histological and statistical approaches herein adopted confirmed that dietary HI meal inclusion did not lead to the development of gut inflammation, but also revealed that the animals fed both the insect-based and the $\mathrm{C}$ diets of the current research displayed greater infiltration with immune cells in the ileum than the other gut segments. This is in agreement with the previously described mucin dynamics in the ileum, thus further underlying the predisposition of this gut segment to be colonized by potential pathogens and, consequently, recall immune cells as defense mechanism. Yu et al. [19] previously observed an up-regulation of the antiinflammatory cytokines and intestinal barrier genes in HI-fed finishing pigs, attributing these changes to an increase in SCFAs-producing bacteria and their metabolites. The parallel identification of SCFAs-producing bacteria and infiltration of immune cells in the gut of the insect-fed piglets of the present study suggests the importance to perform both the histological examination and the gene expression analysis to characterize the intestinal inflammatory status.

\section{Conclusions}

In conclusion, dietary $\mathrm{HI}$ meal inclusion up to $10 \%$ inclusion level may positively modulate the cecal microbiota (in terms of selection of SCFAs-producing bacteria) and the small intestinal mucin composition (in 
terms of stimulation of gut maturity) of the weaned piglets. Furthermore, the histological characterization of the gut infiltration with immune cells highlighted that insect meal utilization has not a significant role in its development. However, further researches performing gene expression analyses for gut mucin and cytokine characterization - as well as metagenomics and metametabolomics for the study of the microbiome - are needed to overcome the above-mentioned limitations and confirm the findings herein observed.

\section{Supplementary information}

Supplementary information accompanies this paper at https://doi.org/10. 1186/s40104-020-00466-X.

Additional file 1: Table S1. Ingredients and chemical composition of the experimental diets. CPremix: $16,000 \mathrm{IU}$ vitamin $\mathrm{A} ; 2,000 \mathrm{IU}$ vitamin $\mathrm{D}_{3}$; $75.0 \mathrm{mg}$ vitamin $\mathrm{E}_{;} 2.94 \mathrm{mg}$ vitamin $\mathrm{K}_{3} ; 3.0 \mathrm{mg}$ vitamin $\mathrm{B}_{1} ; 6.0 \mathrm{mg}$ vitamin $\mathrm{B}_{2} ; 4.0 \mathrm{mg}$ vitamin $\mathrm{B}_{6} ; 0.05 \mathrm{mg}$ vitamin $\mathrm{B}_{12} ; 98 \mathrm{mg}$ vitamin $C_{;} ; 21.0 \mathrm{mg}$ pantothenic acid; $40.0 \mathrm{mg}$ vitamin PP; $1.20 \mathrm{mg}$ folic acid; $0.25 \mathrm{mg}$ biotin; 1500 UI 6-phytase; 700 UI xylanase; 312.5 UI glucanase; 145.68 mg copper; 0.05 mg cobalt; 0.44 mg selenium. HI, Hermetia illucens; DM, dry matter; $\mathrm{CP}$, crude protein; $\mathrm{EE}$, ether extract; NDF, neutral detergent fiber; $A D F$, acid detergent fiber; $\mathrm{NE}$, net energy; $\mathrm{C}=$ control; $\mathrm{HI} 5=5 \%$ inclusion level of Hermetia illucens; HI10 =10\% inclusion level of Hermetia illucens.

Additional file 2: Table S2. Good's coverage and a-diversity measures of cecal microbiota of piglets fed control (C) and 5\% (HI5) and 10\% (HI10) inclusion level of Hermetia illucens meal diets. Description column indicates the 4 replicate boxes per each dietary treatment.

Additional file 3: Table S3. Relative abundance of the main bacterial phyla and genera of cecal microbiota of piglets fed control (C) and 5\% (HI5) and 10\% (HI10) inclusion levels of Hermetia illucens meal diets.

\section{Abbreviations}

CP: Crude protein; DM: Dry matter; EE: Ether extract; FDR: False discovery rate; GALT: Gut-associated lymphoid tissue; gDNA: Genomic DNA; HI: Hermetia illucens; NE: Net energy; OTU: Operational taxonomic unit; PCA: Principal component analysis; SEM: Standard error of the mean; SCFAs: Short chain fatty acids

\section{Acknowledgments}

The authors gratefully acknowledge Mr. Dario Sola, Mr. Mario Colombano and Ms. Alessandra Sereno for technical support and Hermetia Deutschland $\mathrm{GmbH} \& \mathrm{Co}$. KG for the provision of the insect meal.

\section{Authors' contributions}

$I B, M T C, A S, F G$ and $L G$ conceived and designed the experiment. IB, IF, EC, $F G, A S, L C, M T C$ and $L G$ collected the experiments data. IB, W and EC performed the histochemical investigations. IF performed the 16S rRNA amplicon based sequencing. IB and IF performed the statistical analysis. All authors interpreted the data. IB and IF wrote the first draft of the manuscript. All authors critically reviewed the manuscript for intellectual content and gave final approval for the version to be published.

\section{Funding}

Financial support for this work was provided by Martini Group (Premio Iller Campani) and by the University of Turin (ex 60\%) grant (Es. fin. 2015-20162017).

\section{Availability of data and materials}

The datasets analysed in the present study are available from the corresponding author on reasonable request.

\section{Ethics approval and consent to participate}

The experimental protocol was designed according to the guidelines of the current European Directive (2010/63/EU) on the care and protection of animals used for scientific purposes and approved by the Ethical Committee of the Department of Veterinary Sciences of the University of Turin (Italy) (Ref. Ref. 2, 28/06/2016).

\section{Consent for publication}

Not applicable.

\section{Competing interests}

The authors declare that they have no competing interests.

\section{Author details}

${ }^{1}$ Department of Agricultural, Forest and Food Sciences, University of Turin, Largo Paolo Braccini 2, 10095 Grugliasco, TO, Italy. ${ }^{2}$ Department of Veterinary Sciences, University of Turin, Largo Paolo Braccini 2, 10095 Grugliasco, TO, Italy. ${ }^{3}$ Institute of Science of Food Production, National Research Council, Largo Paolo Braccini 2, 10095 Grugliasco, TO, Italy.

Received: 29 January 2020 Accepted: 24 April 2020

Published online: 24 June 2020

\section{References}

1. OECD/FAO. OECD-FAO Agricultural Outlook 2019-2028, OECD publishing, Paris/Food and Agriculture Organization of the United Nations. Rome; 2019. https://doi.org/10.1787/agr_outlook-2019-en. Accessed 05 Dec 2019.

2. McCormack UM, Curião T, Metzler-Zebeli BU, Wilkinson T, Reyer H, Crispie F, et al. Improvement of feed efficiency in pigs through microbial modulation via fecal microbiota transplantation in sows and dietary supplementation of inulin in offspring. Appl Environ Microbiol. 2019;85. https://doi.org/10.1128/ AEM.01255-19

3. Quan J, Cai G, Ye J, Yang M, Ding R, Wang X, et al. A global comparison of the microbiome compositions of three gut locations in commercial pigs with extreme feed conversion ratios. Sci Rep. 2018;8:4536. https://doi.org/10. 1038/s41598-018-22692-0.

4. Celi P, Cowieson AJ, Fru-Nji F, Steinert RE, Kluenter AM, Verlhac V. Gastrointestinal functionality in animal nutrition and health: new opportunities for sustainable animal production. Anim Feed Sci Technol. 2017:234:88-100. https://doi.org/10.1016/j.anifeedsci.2017.09.012.

5. Zhang Z, Tun HM, Li R, Gonzalez BJM, Keenes HC, Nyachoti CM, et al. Impact of xylanases on gut microbiota of growing pigs fed corn- or wheat-based diets. Anim Nutr. 2018:4:339-50. https://doi.org/10.1016/j.aninu.2018.06.007.

6. Desantis S, Mastrodonato M, Accogli G, Rossi G, Crovace AM. Effects of a probiotic on the morphology and mucin composition of pig intestine. Histol Histopathol. 2019;34:1037-50. https://doi.org/10.14670/HH-18-106.

7. Xiao L, Estellé J, Kiilerich P, Ramayo-Caldas Y, Xia Z, Feng Q, et al. A reference gene catalogue of the pig gut microbiome. Nat Microbiol. 2016;1: 16161. https://doi.org/10.1038/nmicrobiol.2016.161

8. Liévin-Le Moal V, Servin AL. The front line of enteric host defense against unwelcome intrusion of harmful microorganisms: mucins, antimicrobial peptides, and microbiota. Clin Microbiol Rev. 2006;19:315-37. https://doi. org/10.1128/CMR.19.2.315-337.2006.

9. Forstner G, Forstner JF. Gastrointestinal mucus. In: Johnson LR, editor. Physiology of the gastrointestinal tract. New York: Raven Press; 1994. p. 1255-84.

10. Wang Y, Yan X, Zhang W, Liu Y, Han D, Teng K, et al. Lactobacillus casei Zhang prevents jejunal epithelial damage to early-weaned piglets induced by Escherichia coli K88 via regulation of intestinal mucosal integrity, tight junction proteins and immune factor expression. J Microbiol Biotechnol. 2019:29:863-76. https://doi.org/10.4014/jmb.1903.03054

11. Meneguz M, Schiavone A, Gai F, Dama A, Lussiana C, Renna M, et al. Effect of rearing substrate on growth performance, waste reduction efficiency and chemical composition of black soldier fly (Hermetia illucens) larvae. J Sci Food Agric. 2018;98:5776-84. https://doi.org/10.1002/jsfa.9127.

12. Gasco L, Finke M, van Huis A. 2018. Can diets containing insects promote animal health? J Insects Food Feed. 2018;4:1-4. https://doi.org/10.3920/JIFF2018.x001.

13. Neumann C, Velten S, Liebert F. N balance studies emphasize the superior protein quality of pig diets at high inclusion level of algae meal (Spirulina platensis) or insect meal (Hermetia illucens) when adequate amino acid supplementation is ensured. Animals. 2018;8:172. https://doi.org/10.3390/ani8100172.

14. Chia SY, Tanga CM, Osuga IM, Alaru AO, Mwangi DM, Githinji M, et al. Effect of dietary replacement of fishmeal by insect meal on growth performance, blood profiles and economics of growing pigs in Kenya. Animals. 2019:9. https://doi.org/10.3390/ani9100705 
15. DiGiacomo K, Leury BJ. Review: insect meal: a future source of protein feed for pigs? Animal. 2019;13:3022-30. https://doi.org/10.1017/ S1751731119001873.

16. Spranghers T, Michiels J, Vrancx J, Ovyn A, Eeckhout M, De Clercq P, et al. Gut antimicrobial effects and nutritional value of black soldier fly (Hermetia illucens L.) prepupae for weaned piglets. Anim Feed Sci Technol. 2018;235: 33-42. https://doi.org/10.1016/j.anifeedsci.2017.08.012.

17. Biasato I, Renna M, Gai F, Dabbou S, Meneguz M, Perona G, et al. Partially defatted black soldier fly lanva meal inclusion in piglet diets: effects on the growth performance, nutrient digestibility, blood profile, gut morphology and histological features. J Anim Sci Biotechnol. 2019;10:12. https:/doi.org/10.1186/s40104-019-0325-x.

18. Regulation (EC) No $999 / 2001$ of the European Parliament and of the Council of 22 May 2001. Laying down rules for the prevention, control and eradication of certain transmissible spongiform encephalopathies. Off J Eur Communities. 2001:L147:1-40.

19. Yu M, Li Z, Chen W, Rong T, Wang G, Ma X. Hermetia illucens larvae as a potential dietary protein source altered the microbiota and modulated mucosal immune status in the colon of finishing pigs. J Anim Sci Biotechnol. 2019;10:50. https://doi.org/10.1186/s40104-019-0358-1.

20. Klindworth A, Pruesse E, Schweer T, Peplies J, Quast C, Horn M, et al. Evaluation of general 16S ribosomal RNA gene PCR primers for classical and next-generation sequencing-based diversity studies. Nucleic Acids Res. 2013; 41:e1. https://doi.org/10.1093/nar/gks808.

21. Rieger J, Drewes B, Hünigen $H$, Plendl J. Mucosubstances in the porcine gastrointestinal tract: fixation, staining and quantification. Eur J Histochem. 2019;63. https://doi.org/10.4081/ejh.2019.3030.

22. Saqui-Salces M, Huang Z, Vila MF, Li J, Mielke JA, Urriola PE, et al. Modulation of intestinal cell differentiation in growing pigs is dependent on the fiber source in the diet. J Anim Sci. 2017;95:1179-90. https://doi.org/10.2527/jas.2016.0947.

23. Magoč T, Salzberg SL. FLASH: fast length adjustment of short reads to improve genome assemblies. Bioinformatics. 2011;27:2957-63. https://doi. org/10.1093/bioinformatics/btr507.

24. Caporaso JG, Kuczynski J, Stombaugh J, Bittinger K, Bushman FD, Costello EK, et al. QIIME allows analysis of high-throughput community sequencing data. Nat Methods. 2010;7:335-6. https://doi.org/10.1038/nmeth.f.303.

25. Biasato I, Ferrocino I, Biasibetti E, Grego E, Dabbou S, Sereno A, et al. Modulation of intestinal microbiota, morphology and mucin composition by dietary insect meal inclusion in free-range chickens. BMC Vet Res. 2018; 14:383. https://doi.org/10.1186/s12917-018-1690-y.

26. Dixon P. VEGAN, a package of $R$ functions for community ecology. J Veg Sci. 2003;14:927-30. https://doi.org/10.1111/j.1654-1103.2003.tb02228.x.

27. Sun Y, Su Y, Zhu W. Microbiome-Metabolome responses in the cecum and colon of pig to a high resistant starch diet. Front Microbiol. 2016;7:779. https://doi.org/10.3389/fmicb.2016.00779

28. Kiros TG, Luise D, Derakhshani H, Petri R, Trevisi P, D'Inca R, et al. Effect of live yeast Saccharomyces cerevisiae supplementation on the performance and cecum microbial profile of suckling piglets. PLoS One. 2019;14: e0219557. https://doi.org/10.1371/journal.pone.0219557.

29. Gao P, Liu Y, Le B, Qin B, Liu M, Zhao Y, et al. A comparison of dynamic distributions of intestinal microbiota between large White and Chinese Shanxi black pigs. Arch Microbiol. 2019;201(3):357-67. https://doi.org/10. 1007/s00203-019-01620-4.

30. Borrelli L, Coretti L, Dipineto L, Bovera F, Menna F, Chiariotti L, et al. Insectbased diet, a promising nutritional source, modulates gut microbiota composition and SCFAs production in laying hens. Sci Rep. 2017;7:16269. https://doi.org/10.1038/s41598-017-16560-6.

31. Biasato I, Ferrocino I, Grego E, Dabbou S, Gai F, Gasco L, et al. Gut microbiota and mucin composition in female broiler chickens fed diets including yellow mealworm (Tenebrio molitor, L.). Animals (Basel). 2019;9. https://doi.org/10.3390/ani9050213

32. Liu C, Finegold SM, Song Y, Lawson PA. Reclassification of Clostridium coccoides, Ruminococcus hansenii, Ruminococcus hydrogenotrophicus, Ruminococcus luti, Ruminococcus productus and Ruminococcus schinkii as Blautia coccoides gen. nov., comb. nov., Blautia hansenii comb. nov., Blautia hydrogenotrophica comb. nov., Blautia luti comb. nov., Blautia producta comb. nov., Blautia schinkii comb. nov. and description of Blautia wexlerae sp. nov., isolated from human faeces. Int J Syst Evol Microbiol. 2008;58: 1896-902. https://doi.org/10.1099/ijs.0.65208-0..

33. Yang L, Liu S, Ding J, Dai R, He C, Xu K, et al. Gut microbiota comicroevolution with selection for host humoral immunity. Front Microbiol. 2017:8:1243. https://doi.org/10.3389/fmicb.2017.01243.
34. Levine UY, Looft T, Allen HK, Stanton TB. Butyrate-producing bacteria, including mucin degraders, from the swine intestinal tract. Appl Environ Microbiol. 2013;79:3879-81. https://doi.org/10.1128/AEM.00589-13.

35. Wu GD, Chen J, Hoffmann C, Bittinger K, Chen YY, Keilbaugh SA, et al. Linking long-term dietary patterns with gut microbial enterotypes. Science. 2011;334:105-8. https://doi.org/10.1126/science.1208344.

36. Accetto T, Auguštin G. Polysaccharide utilization locus and CAZYme genome repertoires reveal diverse ecological adaptation of Prevotella species. Syst Appl Microbiol. 2015;38:453-61. https://doi.org/10.1016/j.syapm.2015.07.007.

37. Fang $S$, Xiong $X$, Su Y, Huang $L$, Chen C. $16 S$ rRNA genebased association study identified microbial taxa associated with pork intramuscular fat content in feces and cecum lumen. BMC Microbiol. 2017;17:162. https://doi. org/10.1186/s12866-017-1055-x.

38. Scott KP, Martin JC, Chassard C, Clerget M, Potrykus J, Campbell G, et al. Substrate-driven gene expression in Roseburia inulinivorans: importance of inducible enzymes in the utilization of inulin and starch. Proc Natl Acad Sci U S A. 2011;108:4672-9. https://doi.org/10.1073/pnas.1000091107.

39. David LA, Maurice CF, Carmody RN, Gootenberg DB, Button JE, Wolfe BE, et al. Diet rapidly and reproducibly alters the human gut microbiome. Nature. 2014;505:559-63. https://doi.org/10.1038/nature12820.

40. Salonen A, Lahti L, Salojärvi J, Holtrop G, Korpela K, Duncan SH, et al. Impact of diet and individual variation on intestinal microbiota composition and fermentation products in obese men. ISME J. 2014;8:2218-30. https://doi. org/10.1038/ismej.2014.63.

41. Flint HJ, Bayer E, Rincon M, Lamed R, White BA. Polysaccharide utilization by gut bacteria: potential for new insights from genomic analysis. Nat Rev Microbiol. 2008;6:121-31. https://doi.org/10.1038/nrmicro1817.

42. Chassard C, Delmas E, Robert C, Lawson PA, Bernalier-Donadille A. Ruminococcus champanellensis sp. nov., a cellulose-degrading bacterium from human gut microbiota. Int J Syst Evol Microbiol. 2012;62:138-43. https://doi.org/10.1099/ijs.0.027375-0.

43. Roy $C C$, Kien $C L$, Bouthillier $L$, Levy $E$. Short-chain fatty acids: ready for prime time? Nutr Clin Pract. 2006;21:351-66. https://doi.org/10.1177/ 0115426506021004351

44. Flint HJ, Scott KP, Louis P, Duncan SH. The role of the gut microbiota in nutrition and health. Nat Rev Gastroenterol Hepatol. 2012;9:577. https://doi. org/10.1038/nrgastro.2012.156.

45. Louis P, Hold GL, Flint HJ. The gut microbiota, bacterial metabolites and colorectal cancer. Nat Rev Microbiol. 2014;12:661. https://doi.org/10.1038/nrmicro3344.

46. Lallès JP, Boudry G, Favier C, Le Floc'h N, Luron I, Montagne L. Gut function and dysfunction in young pigs: physiology. Anim Res. 2004;53:301-16. https://doi.org/10.1051/animres:2004018.

47. Zapata DJ, Rodríguez BJ, Ramírez MC, López A, Parra J. Escherichia coli lipopolysaccharide affects intestinal mucin secretion in weaned pigs. Rev Colomb Cienc Pecu. 2015;28:209-17.

48. Turck D, Feste A, Lifschitz C. Age and diet affect the composition of porcine colonic mucins. Pediatr Res. 1993;33:564-7. https://doi.org/10.1203/ 00006450-199306000-00005.

49. Law GK, Bertolo RF, Adjiri-Awere A, Pencharz PB, Ball RO. Adequate oral threonine is critical for mucin production and gut function in neonatal piglets. Am J Physiol Gastrointest Liver Physiol. 2007;292:G1293-301.

50. Forder RE, Howarth GS, Tivey DR, Hughes RJ. Bacterial modulation of small intestinal goblet cells and mucin composition during early posthatch development of poultry. Poult Sci. 2007;86:2396-403. https://doi.org/10. 3382/ps.2007-00222

51. Brown PJ, Miller BG, Stokes CR, Blazquez NB, Bourne FJ. Histochemistry of mucins of pig intestinal secretory epithelial cells before and after weaning. J Comp Pathol. 1988;98:313-23. https://doi.org/10.1016/0021-9975(88)90040-0.

52. Georgiades P, Pudney PD, Thornton DJ, Waigh TA. Particle tracking microrheology of purified gastrointestinal mucins. Biopolymers. 2014;101: 366-77. https://doi.org/10.1002/bip.22372.

53. Runnels PL, Moon HW, Schneider RA. Development of resistance with host age to adhesion of K99+ Escherichia coli to isolated intestinal epithelial cells. Infect Immun. 1980;28:298-300.

54. Dean-Nystrom EA, Samuel JE. Age-related resistance to 987P fimbriamediated colonization correlates with specific glycolipid receptors in intestinal mucus in swine. Infect Immun. 1994;62:4789-94. 